Volume 9, No.1.3, 2020

International Journal of Advanced Trends in Computer Science and Engineering

Available Online at http://www.warse.org/IJATCSE/static/pdf/file/ijatcse4691.32020.pdf

https://doi.org/10.30534/ijatcse/2020/4691.32020

\title{
A Secure Cloud based Financial Market Research System using Big Data Analytics
}

\author{
Fafeena mole H. ${ }^{1}$, Parvathy R. ${ }^{2}$, P. Mohammed Shameem ${ }^{3}$ \\ ${ }^{1}$ PG Scholar, Department of Computer Science and Engineering, TKMIT fafeeh@gmail.com \\ ${ }^{2}$ Assistant Professor, Department of Computer Science and Engineering, TKMIT toparvathyr@ gmail.com \\ ${ }^{3}$ Professor, Department of Computer Science and Engineering, TKMIT pms.tkmit@yahoo.in
}

\begin{abstract}
Mathematical finance is the application of mathematical methods to financial problems. Cloud based processing of market analytics has been contributing to the potential growth and stability of financial markets. Enhanced performance by the employment of parallel processing algorithms along with the mathematical models to inspect various patterns that either boost or splash the trends in trade have accelerated the research scope in Financial Informatics. The proposed system is a big data infrastructure to project the huge volume of real-time data sequences to predictions through multipart parallel streaming. For that purpose, the proposed concept integrate a prediction module to the existing QuantCloud architecture. Encrypted data processing with customized attributes and record sets is an added advantage of the proposed system. In order to improve the number of responses distributed parallel computing strategies driven by the data itself are used. Experimental results show that the system provides high response rate in providing fastest assistance by means of crunching an aggregation technique. The optimization techniques have resulted in reduced latency. A comparative study conducted with state-of-the-art financial engineering tools that provide the services proved that the proposed method provide better results in terms of throughput, security and trustworthiness.
\end{abstract}

Key words : Big Data, Cloud computing, Quantitative finance, parallel processing.

\section{INTRODUCTION}

Information technology has spread its wings to almost all disciplines with the widespread use of web-enabled services. This service dissemination required research in developing best-fit mathematical models for analyzing the large volume of data evolving in the user community. In the finance industry, which mainly refers to stock markets, the data to be processed real-time is so huge that measured in zeta bytes or even more. As the financial industry rapidly moves toward data on-demand optimization, companies must respond to these changes in a deliberate and comprehensive manner. Quantitative finance deals with the design and use of mathematical models and extremely large datasets to analyze financial markets and allied industries and associated risks. A quantitative analyst uses mathematical models and applies them to financial markets in order to support the trading and risk management departments that operate in banks and financial institutions. The industry uses a new jargon to address the professionals who work in this field, namely, Quants. Quantitative finance is now opening a new discipline of financial mathematics which deals with the design and application of such models and derivatives.

Mathematical finance is a field that replies on mathematic models and massive market data to provide the actionable, quantitative information for all aspects of the finance [1], [2], [3], [4]. It draws on tools from probability, statistics, stochastic processes, and economic theory. Financial mathematics was traditionally applied to such problems as derivative security valuation, portfolio structuring, risk management, and scenario simulation.

Understanding market data is vital for understanding the financial market in the big data era [3]. In the finance industry the data is no longer measured in terabytes but in zeta bytes or even more. As the financial industry rapidly moves toward data on-demand optimization, companies must respond to these changes in a deliberate and comprehensive manner. Substantial and important research has been undertaken in risk and financial management that is related to big data, Economics, Finance, Marketing and Management. Big data in finance refers to the petabytes of structured and unstructured data that can be used to anticipate customer behaviors and create strategies for banks and financial institutions. The finance industry generates lots of data. Structured data is information managed within an organization in order to provide key decision-making insights. Unstructured data exists in multiple sources in increasing volumes and offers significant analytical opportunities. 
Fafeena mole H et al., International Journal of Advanced Trends in Computer Science and Engineering, 9(1.3), 2020,303 - 309

Algorithmic trading [5] uses vast historical data with complex mathematical models in conjunction with big data to maximize portfolio returns. Capital markets need to actively manage historical data as well as real-time data from stock tickers. Extracting the insightful information for profitable signals is of first priority to the algorithmic traders. The automated process enables computer programs to execute financial trades at speeds and frequencies that a human trader cannot. Within the mathematical models, algorithmic trading provides trades executed at the best possible prices and timely trade placement and reduces manual errors due to behavioral factors. Algorithms based on economic theory typically point to long-term investment opportunities due to trends in historical data. Efficiently producing results supporting a short-term investment strategy is the inherent challenges in predictive models. In other words, more efficient big data infrastructure can create profitable opportunities.

Financial data analysis in algorithmic trading is carried out in various phases. The primary operations covered in these are mainly first data cleaning and aggregating, and then data modelling based on the cleaned grouped data. Data cleaning is the process of preparing data for analysis by removing or modifying data that is incorrect, incomplete, irrelevant, duplicated, or improperly formatted. Following this, aggregating data is to yield statistics of data with the desired frequency. The next task is data modelling where sophisticated analytics is constructed to extract the signals from the aggregated, grouped data. For example, the machine learning method could be designed to analyze big data [6].

In the view of the data-crunching need, financial firms never stop seeking a scalable one-stop solution that covers all aspects. Inspired by this need, we design and develop an integrated platform by combining a big data infrastructure and a data on-demand execution model. This integrated platform is referred to as the QuantCloud platform. Essentially, QuantCloud executes the complex event processing in a data on-demand mode and manages large amounts of varying market data in a data-parallel model. More importantly, the results of our tests demonstrated an achieved performance record: a high throughput and a sub-microsecond latency, and a support for multiple data-dependent tasks in multicore architectures.

Stock price prediction has been a focus for years since it can yield significant profits. Predicting the stock market is not a simple task, mainly as a consequence of the close to random-walk behavior of a stock time series. Fundamental and technical analyses were the first two methods used to forecast stock prices. Artificial Neural networks (ANNs) is the most commonly used technique. In most cases, ANNs suffer from the over-fitting problem. Due to the large number of parameters to fix, and the little prior user knowledge about the relevance of the inputs in the analyzed problem. Also, Support vector machines (SVMs) had been developed as an alternative that avoids such limitations. Their practical successes can be attributed to solid theoretical foundations based on VC-theory. SVM compute globally optimal solutions, unlike those obtained with ANNs, which tend to fall into local minima.

Machine learning is an application of Artificial Intelligence (AI) that provides systems with the ability to automatically learn and improve from experience without being explicitly programmed. Machine learning is using data to train a model and then using the trained model to make predictions from new data. The fundamental machine learning problem is to find a method able to learn the complex features of the data inputs which lead to good predictions of the target output variables, and a predictor of an output $\mathrm{Y}$ given an input $\mathrm{X}$.

The rest of paper is organized as follows: Section 2 presents the related work followed by the overview of QuantCloud architecture in Section 3. Section 4 presents the methodology. Section 5 Results are presented and Conclusion is drawn in Section 6.

\section{RELATED WORK}

The inherent advantages of cloud computing including scalability, availability, usability, low lifetime costs attract financial enterprises instead of sitting up an on-premise computing environment. Fenu. G \& Surcis. S [7] has designed a real-time financial system based on cloud computing technologies that enable macroeconomic analysis and forecasts of the financial markets and their instruments.

P. Zhang, K. Yu, J. Yu, and S. Khan developed the QuantCloud infrastructure [8] for big data analytics in modern quantitative finance on a 40-core, 1TB-memory computer and a 5-TB Solid State Drives (SSD)-backed datastore, using NYSE TAQ data from the fourth quarter of 2014 as our market data. They have employed large-scale SSD-backed datastore, various parallel processing algorithms, and portability in Cloud computing. QuantCloud bridges the gap between model computing techniques and financial data-driven research. The large volume of market data is structured in an SSD-backed datastore, and a program is run in the background which reacts to provide the Data-on-Demand services. Multiple client services process user requests in a parallel mode and query on-demand datasets from the datastore through Internet connections. The results indicate that the aggregated contributions of infrastructure, parallel algorithms, and sophisticated implementations offer the algorithmic trading and financial engineering community new hope and numeric insights for their research and development. 
Fafeena mole H et al., International Journal of Advanced Trends in Computer Science and Engineering, 9(1.3), 2020,303 - 309

Quantitative finance had hype in the last decade which was dependent on computer techniques that make processing large datasets possible. As more data is available at a higher frequency, more researches in quantitative finance have switched to the microstructures of the financial market. High-frequency data is a typical example of big data that is characterized by velocity, variety and volume. In addition, the signal-to-noise the ratio in financial time series is usually very small. High-frequency datasets are more likely to be exposed to extreme values, jumps and errors than the low-frequency ones. Specific data processing techniques and quantitative models are elaborately designed to extract information from financial data efficiently. An exhaustive review of the revolutionary changes that big data analytics using computational means has brought in the financial industry has been published by B. Fang and P. Zhang [3]. They point out the scope of quantitative tools for addressing massive financial data. Adoptions of advanced statistics, information theory, machine learning and faster computing algorithms are inevitable in order to predict complicated financial markets.

X. Shi, P. Zhang, and S. U. Khan [4] highlights the need for a new platform from the business philosophy, business model, technical means, sales, internal management and other dimensions to re-shape the financial industry. They have combined the existing big data platform architecture technology innovation and space-time data elements with the insurance industry for practical analysis.

I. A. T. Hashem, I. Yaqoob, N. B. Anuar, S. Mokhtar, A. Gani, and S. U. Khan [9] discuss the effective use of cloud computing in the development of big data infrastructure. Cloud computing offers access to data storage, processing, and analytics on a more scalable, flexible, cost-effective, and even secure basis than can be achieved with an on-premises deployment. These characteristics are essential for customers when data volumes are growing exponentially to make storage and processing resources available as needed, as well as to get value from that data. The cloud offers a way to experiment with managed services in a pay-as-you-go manner. The cloud computing paradigm allows users to quickly deploy on-demand applications on converged infrastructure and shared services [10]. In this regard, it can provide scalable computing resources while lowering operational costs, drawing attention to financial computing service.

J. Chevalier and G. Ellison [11] presents an analytical study of the demand for a market-wide risk management system that would deal with computer-generated chaos in real time is raised. R. A. Brealey and E. Kaplanis [12] substantiates that providing cloud services to major financial institutions is no longer so much about the arguments for or against any particular cloud model. Instead, it's about changing culture. Smaller companies are willing to pay on demand for cloud services, but the bigger ones are not. Banks can use the cloud to store and analyses big data, and we have the technology to be secure. What's lagging behind is the culture of conservatism at the big banks. How to juggle increasing demands from consumers and regulators against decreased resources and shrinking margins remains as a key challenge. Extensive research has been conducted in using machine learning techniques for financial data analytics even before the widespread use of big data. Usmani, Mehak, Syed Hasan Adil, Kamran Raza, and Syed Saad Azhar Ali [13] proposes a method for stock market prediction using machine learning techniques. The experimental results show that the Multi-Layer Perceptron algorithm of machine learning predicted $77 \%$ correct market performance. Even with the lack of resources and unavailability of data for the market, the model was able to predict the performance to a good extent with only 100 instances.

\section{QUANTCLOUD ARHITECTURE}

QuantCloud consists of (i) a database system (referred to as Server), (ii) a parallel computing platform (referred to as Client), and (iii) a cloud portal (referred to as User) as illustrated in Figure 1.

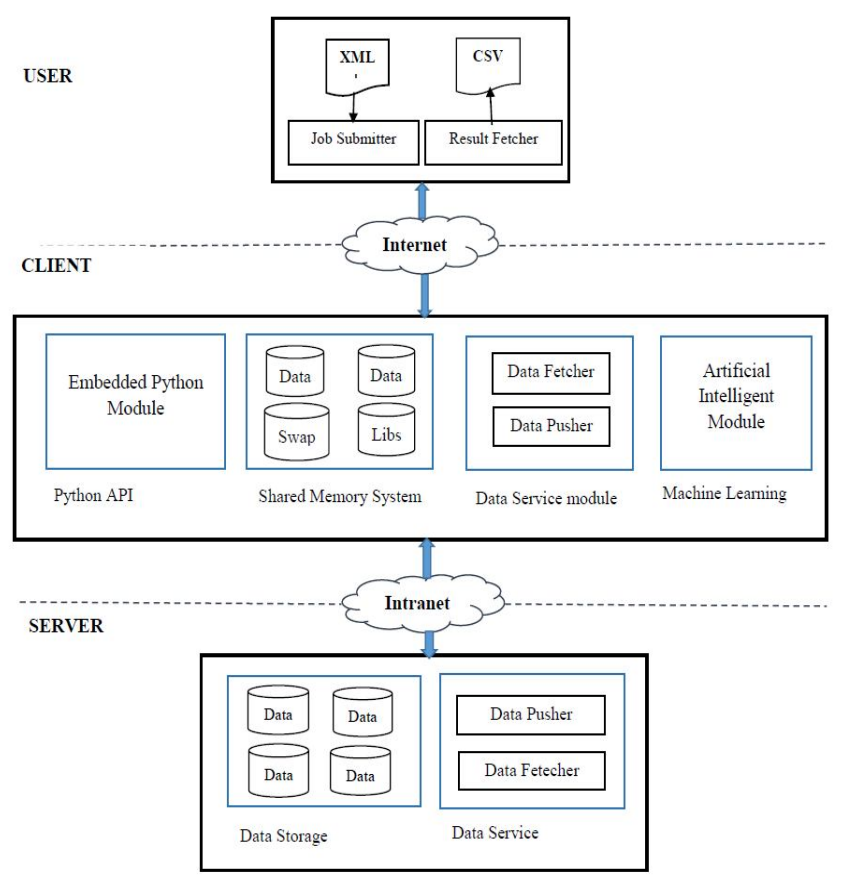

Figure 1: Overview of QuantCloud Architecture

The Server part manages the market data as compressed and hashed time series data. Server permits data storage and data query services and it employs a client-server model as a distributed application structure that partitions queries 
Fafeena mole $\mathrm{H}$ et al., International Journal of Advanced Trends in Computer Science and Engineering, 9(1.3), 2020,303 - 309

between the data provider (Server) and the data requesters (Client). The User part is a portal through which an end-user submits an algorithm and parameters as a task. The task is received and executed on the Client. The Server provides data to Client and Client executes the algorithm on provided data - this response to a task request from the User. In this architecture, the Server resides on a Storage-As-a-Service (SaaS) platform while the Client is managed on an Infrastructure-As-a-Service (IaaS) platform that provides an instant computing infrastructure over the Internet.

\section{METHODOLOGY}

In the present work, proposes the integration of a prediction module to the existing QuantCloud architecture. The newly incorporated module is assumed to give a time series forecast of the stock data. Since the target is big data, we apply deep learning algorithms to frame the prediction module. An overview of the proposed market prediction module is given in Figure 2.

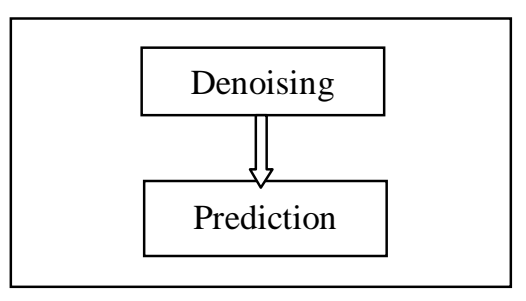

Figure 2: Prediction module

\subsection{Denoising}

It has been considered that the presence of a high degree of noise is a key factor that challenges the predictions in case of stock price data [14]. Thus denoising remains as the primary step in the forecasting of stock price data. For denoising, we apply the wavelet transform method. A wavelet transform decomposes a signal into different scales, making it possible to analyze and extract important information contained in noisy signals [15]. Thus it is a widely used tool for the denoising of stock market data. [16], [17], [18], [19].

Wavelet transform allows processing data at different scales by decomposing it into many frequency bands. The ability of wavelet transform to analyses frequency and time domain simultaneously is utilized widely in analyzing time-varying like the stock market data [20]. Wavelets allow to trade off the time and frequency resolution in different ways. It allows viewing different parts of the data through different sized windows in order to suit the need. Thus the wavelet transform allows to represent the data at various multiresolution levels, each coefficient sets being processed in order to remove the less significant information. Thus wavelet analysis allows us to extract the important hidden information and significant temporal features of the original time series data [21]. This enables to filter the noise components in the original data.
In wavelet transform, the signal $o(t)$ is decomposed into smooth coefficients $\mathrm{A}$ and detail coefficients $\mathrm{D}$, which are represented by,

$$
\begin{aligned}
& A_{i_{i j}}=\int a(t) \Phi_{i_{i j}}(t) d t \\
& D_{i_{j}}=\int a(t) \Psi_{i_{j}}(t) d t
\end{aligned}
$$

Here $\Phi$ represents the father wavelet and $\Psi$ represents mother wavelets, $i$ and $j$ denote the scaling and translation. The father wavelet approximates the approximation coefficients and the mother wavelet approximates the detailed coefficients and is defined as follows:

$$
\begin{aligned}
& \Phi_{i, j}(t)=2^{-i / 2} क\left(2^{-i} j-j\right) \\
& \Psi_{i, j}(t)=2^{-i / 2} \Psi\left(2^{-i j}-j\right)
\end{aligned}
$$

Where

$$
\begin{aligned}
& \int \Phi(t) d t=1 \\
& \int \Psi(t) d t=0
\end{aligned}
$$

Therefore the orthogonal wavelet representation of the original signal $a(t)$ is defined by

$$
\begin{aligned}
o(t)=\sum_{j} a_{i, j} \dot{\phi}_{i j}(t) & +\sum_{j} d_{i j} \Psi_{i, j}(t) \\
& +\sum_{j} a_{i-1, j} \Psi_{i-1, j}(t)+\ldots+\sum_{j} a_{i j j} \Psi_{i, j}(t)
\end{aligned}
$$

There are different types of wavelets can be used and in our work, Haar wavelet is applied.

\subsection{Prediction}

For prediction, the deep learning architecture- Long-Short Term Memory (LSTM) - is selected as it is capable of handling time series data. LSTM is a kind of Recurrent Neural Network (RNN), a type of neural network. RNN is popular as it holds memory in the Hidden layer. Same parameters are used for each input so that the computations on all the inputs or hidden layers remain the same. However, RNN has the limitation of the gradient being vanished if the input series is too long. LSTM was developed in order to overcome this problem in Recurrent Neural Networks [22]. LSTM possess memory which passes its state to the next state without matrix multiplication and operation of activation function, so the gradient is back-propagated smoothly [23]. This makes LSTM as a popular choice for the stock market prediction problems [24], [25], [26], [27][29].

Long-Short Term Memory: Figure 3 gives the architecture of a single cell among the repeating memory cells in the LSTM network [28]. 


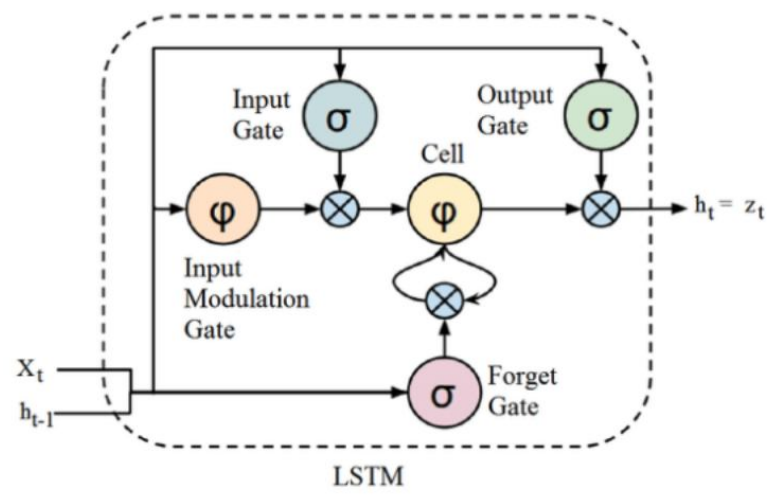

Figure 3: Architecture of a single cell LSTM network

The components of the model are:

- Forget gate $(f)$ : The initial step of processing through LSTM is done by the forget gate which decides which information to forget from the cell state. This is done by implementing a sigmoid activation function on $\mathrm{X}_{\mathrm{t}}$ and $\mathrm{h}_{\mathrm{t}-1}$. The information is removed in the cell state if the output is 0 .

$$
f_{t}=\sigma\left(W_{f},\left\lfloor h_{t-1}, x_{t}\right\rfloor+b_{f}\right)
$$

- Input gate $\left(i_{i}\right)$ : Decides what information from current input should be stored in the cell state. The activation function for the input gate is a sigmoid function. Next, a tanh layer is placed which creates a vector of new cell state values $\left(C_{t}\right)$ that is added to the state. Both these values are then used to update the state.

$$
\begin{gathered}
i_{t}=\sigma\left(W_{i} \cdot\left[h_{t-1} x_{t}\right]+b_{i}\right) \\
\zeta_{t}=\tanh \left(W_{c} \cdot\left[h_{t-1}, x_{t}\right]+b_{c}\right)
\end{gathered}
$$

- Cell state $\left(C_{\mathrm{t}}\right)$ : This represents the internal memory of the cell which stores both short term memory and long-term memories. Updating $C_{t-1}$ to the new cell state $C_{\mathrm{I}}$ requires multiplying the old state by $f$. Then the new candidate values are added, scaled by the value from the input gate.

$$
C_{t}=f_{t} * C_{t-1}+i_{t} * \widetilde{C}_{t}
$$

- Output gate $\left(a_{t}\right)$ : Decides what information from the current cell state flows into the hidden state using a sigmoid function.

$$
o_{t}=\sigma\left(W_{o}\left[h_{t-1}, x_{t}\right]+b_{0}\right)
$$

- Hidden state $\left(\mathrm{h}_{\mathrm{t}}\right)$ : This is the working memory which decides which information should be taken to the next sequence. The tanh function is applied on the cell state values to get the values between -1 and 1 which is then multiplied by the output of the sigmoid gate of the hidden state. This will enable to output only the desired values.

$$
h_{t}=o_{t} * \tanh \left(C_{t}\right)
$$

\subsection{Dataset}

This work mainly uses the data extracted from Google stocks, Yahoo Finance and Twitter for testing the algorithms. The tweets are collected using the search API provided by Twitter. The search API allows fine-tuning queries filtering based on time, region etc. in recent tweets.

The yahoo API is used to collect stock related news from the yahoo finance website. Here the URL of the news feeds are gathered and fetch them as a batch file. The extracted data are passed as a plain text from the yahoo feed to our proposed system. Yahoo provides the RSS feeds for each news content, they are lightweight and easy to process.

Screenshot of the data fetching process is given in figure 4 .

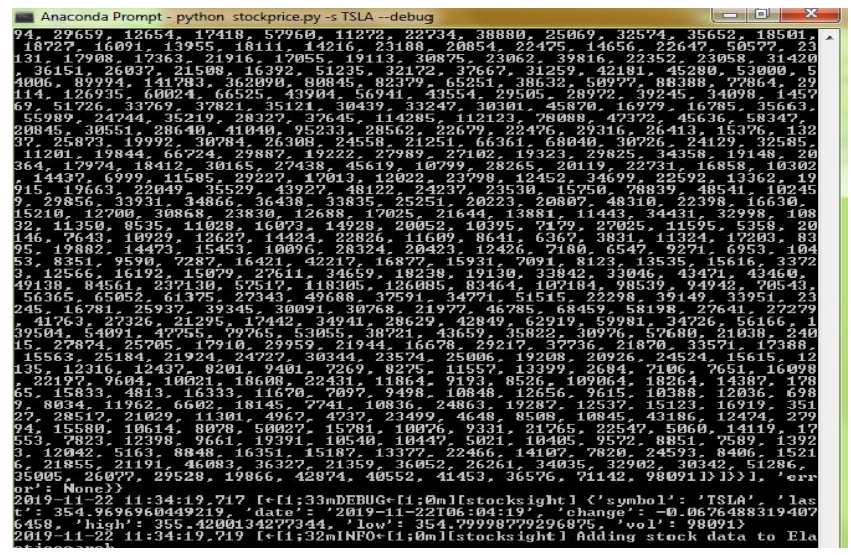

Figure 4: Data fetching process

The yahoo API is used to collect stock related news from the yahoo finance website. Here the URL of the news feeds are gathered and fetch them as a batch file. The extracted data are passed as a plain text from the yahoo feed to our proposed system. Yahoo provides the RSS feeds for each news content, they are lightweight and easy to process.

\section{RESULTS}

Using the LSTM model developed for the stock market trend prediction. The predicted results are plotted against the actual trend to enable comparison. The following table gives a sample set of information summarized from the predictions. 
Fafeena mole $\mathrm{H}$ et al., International Journal of Advanced Trends in Computer Science and Engineering, 9(1.3), 2020,303 - 309

Table 1: Sample set of predictions

\begin{tabular}{|l|l|l|l|}
\hline $\begin{array}{c}\text { Test } \\
\text { Case }\end{array}$ & \multicolumn{1}{|c|}{ Unit } & \multicolumn{1}{|c|}{ Expected } & \multicolumn{1}{|c|}{ Actual } \\
\hline 1 & ITC & Fall by $5 \%$ & Fall by $5 \%$ \\
\hline 2 & WIPRO & Rise by $9 \%$ & Rise by $8.5 \%$ \\
\hline 3 & TSLA & Fall by $4 \%$ & Fall by $4 \%$ \\
\hline 4 & HDFC & Rise by $5 \%$ & Rise by $6 \%$ \\
\hline 5 & SAIL & Rise by $7 \%$ & Rise by $7 \%$ \\
\hline 6 & BPCL & Rise by $6 \%$ & Rise by $6 \%$ \\
\hline 7 & DLF & Fall by $8 \%$ & Fall by $8 \%$ \\
\hline 8 & NTPC & Fall by $5 \%$ & Fall by $5 \%$ \\
\hline
\end{tabular}

After authenticating using a key, we were able to access through python library called Tweepy. The text of each tweet contains too many extraneous words that do not consider its sentiment. Tweets include URLs, tags to others and many other symbols that do not have any sentimental value. We have used python's Natural Language Toolkit (NLT) library to remove stopwords. Stopwords contain articles, punctuation and some extra words which do not have any sentimental value. There is a stop word dictionary that checks each word in a list of tokenized words against a dictionary. If the word is stopword then it is filtered out.

The accuracy of the system is measured as the percentage of the predictions that were correctly determined by the system. For instance, if the system forecasts an upward trend and the index indeed goes up, it is supposed to be correct, otherwise, if the index goes down or remains stable for an uptrend, it is assumed to be wrong. The results show that the proposed method is effective and reliable in making accurate predictions.

\section{CONCLUSION}

In the presented work, we have integrated the prediction module to the data cleaning, aggregating and modelling services offered by the QuantCloud architecture. Existing QuantCloud architecture has already been highlighted due to its ability to fulfill the need for big data complex event processing. The system could successfully explore a huge volume of data from various sources and may predictions accordingly. The integration of the prediction module complements the scope of the architecture as forecasting is of wide demand in the field of quantitative finance when considering the state of the art.

The market predictions can be further improved by making use of an interpolated estimate of the performance of the company in recent past. More research can be conducted in future on the scope of incorporating additional parameters like the influence of market fluctuations and environmental issues in the business balancing of the firm.

\section{REFERENCES}

1. W. H€ardle, T. Kleinow, and G. Stahl, Applied Quantitative Finance. Berlin, Germany: Springer, 2002. https://doi.org/10.1007/978-3-662-05021-7

2. E. Thorp, A perspective on quantitative finance: Models for beating the market, The Best of Wilmott. Hoboken, NJ, USA: Wiley, 2005.

3. B. Fang and P. Zhang, Big data in finance, in Big Data Concepts, Theories, and Applications. S. Yu and S. Guo, Eds., Cham, Switzerland: Springer, 2016, pp. 391-412.

4. X. Shi, P. Zhang, and S. U. Khan, Quantitative data analysis in finance, in Handbook of Big Data Technologies. A. Y. Zomaya and S. Sakr, Eds., Cham, Switzerland: Springer, 2017, pp. 719-753.

5. A. P. Chaboud, B. Chiquoine, E. Hjalmarsson, and C. Vega, Rise of the machines: Algorithmic trading in the foreign exchange market, J. Finance, vol. 69, pp. 2045-2084, 2014. https://doi.org/10.1111/jofi.12186

6. M. Abadi, P. Barham, J. Chen, Z. Chen, A. Davis, J. Dean, et al., TensorFlow: A system for large-scale machine learning, in Proc. OSDI, 2016, pp. 265-283.

7. Fenu, G., and Surcis, S. A cloud computing based real time financial system. In 2009 Eighth International Conference on Networks, IEEE pp. 374-379. March, 2009.

8. P. Zhang, K. Yu, J. Yu, and S. Khan, QuantCloud: Big data infrastructure for quantitative finance on the cloud, IEEE Trans. Big Data doi: 10.1109/TBDATA.2017.2649544, 2017.

9. I. A. T. Hashem, I. Yaqoob, N. B. Anuar, S. Mokhtar, A. Gani, and S. U. Khan, The rise of "big data" on cloud computing: Review and open research issues, Inf. Syst., vol. 47, pp. 98-115, 2015. https://doi.org/10.1016/j.is.2014.07.006

10. D. Kliazovich, P. Bouvry, and S. U. Khan, GreenCloud: A packetlevel simulator of energy-aware cloud computing data centers, J. Supercomput., vol. 62, pp. 1263-1283, 2012.

11. J. Chevalier and G. Ellison, Risk taking by mutual funds as a response to incentives, J. Political Economy, vol. 105, pp. 1167-1200, 1997.

12. R. A. Brealey and E. Kaplanis, Hedge funds and financial stability: An analysis of their factor exposures, Int. Finance, vol. 4, pp. 161-187, 2001.

13. Usmani, Mehak, Syed Hasan Adil, Kamran Raza, and Syed Saad Azhar Ali. Stock market prediction using machine learning techniques. in 20163 rd International Conference on Computer and Information Sciences (ICCOINS), IEEE. pp. 322-327. 2016.

14. Wang, B., Huang, H., \& Wang, X. A novel text mining approach to financial time series forecasting. Neurocomputing, 83, 136-145. 2012. https://doi.org/10.1016/j.neucom.2011.12.013 
15. Cocianu C. L., Grigoryan, H., \& Uscatu, C. Stock Market Forecasting Using Wavelet Denoising Technique and Support Vector Machines.

16. Gupta, S., Wang, L. P., Pedrycz, W., \& Chen, S. M. Neural networks and wavelet de-noising for stock trading and prediction. Time Series Analysis. 2012.

17. Zhao, Y., Zhang, Y., \& Qi, C. Prediction model of stock market returns based on wavelet neural network. In 2008 IEEE Pacific-Asia Workshop on Computational Intelligence and Industrial Application. IEEE. Vol. 1. pp. 31-36. December 2008.

18. Kao, L. J., Chiu, C. C., Lu, C. J., \& Chang, C. H. A hybrid approach by integrating wavelet-based feature extraction with MARS and SVR for stock index forecasting. Decision Support Systems, Vol. 54. No.3, 2013, pp. 1228-1244.

19. Hsieh, T. J., Hsiao, H. F., \& Yeh, W. C. Forecasting stock markets using wavelet transforms and recurrent neural networks: An integrated system based on artificial bee colony algorithm. Applied soft computing, vol.11. No. 2. 2011. pp.2510-2525.

20. Hochreiter, S., \& Schmidhuber, J. Long short-term memory. Neural computation, vol. 9. No. 8, 1997, pp.1735-1780.

21. Chandar, S. K., Sumathi, M., \& Sivanandam, S. N. Prediction of stock market price using hybrid of wavelet transform and artificial neural network. Indian journal of Science and Technology,vol. 9. No. 8, pp. 1-5. 2016.

https://doi.org/10.17485/ijst/2016/v9i8/87905

22. Daubechies, I. The wavelet transform, time-frequency localization and signal analysis. IEEE transactions on information theory, vol. 36. No. 5, pp. 961-1005. 1990.

23. Liu, J., Chao, F., Lin, Y. C., \& Lin, C. M. Stock Prices Prediction using Deep Learning Models. arXiv preprint arXiv:1909.12227. 2019

24. Nelson, D. M., Pereira, A. C., \& de Oliveira, R. A. Stock market's price movement prediction with LSTM neural networks. In 2017 International joint conference on neural networks (IJCNN). IEEE. pp. 1419-1426. May, 2017.

25. Qiu, J., Wang, B., \& Zhou, C. Forecasting stock prices with long-short term memory neural network based on attention mechanism. PloS one, vol. 15. No. 1. 2020. https://doi.org/10.1371/journal.pone.0227222

26. Jiang, Q., Tang, C., Chen, C., Wang, X., \& Huang, Q. Stock price forecast based on LSTM neural network. In International Conference on Management Science and Engineering Management. Springer, Cham. pp. 393-408. August, 2018.

27. Zhang, R., Yuan, Z., \& Shao, X. A New Combined CNN-RNN Model for Sector Stock Price Analysis. In 2018 IEEE 42nd Annual Computer Software and Applications Conference (COMPSAC). IEEE. Vol. 2, pp. 546-551. July, 2018.

https://doi.org/10.1109/COMPSAC.2018.10292
28. Eugine Kang, Long Short-Term Memory (LSTM): Concept, https://medium.com/@kangeugine/longshort- term-memory-lstm-concept-cb3283934359. September, 2017.

29. Vinodh P Vijayan, Biju Paul " Traffic scheduling for Green city through energy efficient Wireless sensor Networks" International Journal of Advanced Trends in Computer Science and Engineering, Volume 8, No.4, July - August 2019, ISSN 2278-3091, https://doi.org/10.30534/ijatcse/2019/81842019. 